\title{
BLACK HOLES, MARGINALLY TRAPPED SURFACES AND QUASI-MINIMAL SURFACES
}

\author{
BANG-YEN CHEN
}

To 60th anniversary of Tamkang University

\begin{abstract}
The concept of trapped surfaces introduced by Sir Roger Penrose in [Phys. Rev. Lett. 14 (1965), 57-59] plays an extremely important role in cosmology and general relativity. A black hole is a trapped region in a space-time enclosed by a marginally trapped surface. In term of mean curvature vector, a space-like surface in a space-time is marginally trapped if its mean curvature vector field is light-like at each point. In this article, we survey recent classification results on marginally trapped surfaces from differential geometric viewpoint. Also, we survey recent results on a closely related subject; namely, quasi-minimal surfaces in pseudoRiemannian manifolds.
\end{abstract}

\section{Black holes and galaxies.}

The idea of an object with gravity strong enough to prevent light from escaping was proposed in 1783 by J. Michell (1724-1793), an amateur British astronomer. P.-S. Laplace (1749-1823), a French physicist came to the same conclusion in 1795 independently. Black holes, as currently understood, are described by Einstein's general theory of relativity developed in 1916 (cf. [26]).

Einstein's theory of general relativity predicts that when a large enough amount of mass is present in a sufficiently small region of space, all paths through space are warped inwards towards the center of the volume, preventing all matter and radiation within it from escaping. Einstein's theory has important astrophysical applications. It points towards the existence of black holes. In addition, general relativity is the basis of current cosmological models of an expanding universe.

According to the American Astronomical Society, every large galaxy has a super massive black hole $\left(\sim 10^{5}-10^{9} M_{\text {sun }}\right)$ at its center. The black hole's mass is proportional to the mass of the host galaxy, suggesting that the two are linked very closely.

Corresponding author: Bang-Yen Chen.

2000 Mathematics Subject Classification. Primary 53C50; 83-02, Secondary 53C40.

Key words and phrases. Trapped surface, black hole, marginally trapped surface, quasi-minimal surface, space-time, Lagangian surface, slant surface. 
Black holes can't be seen, because everything that falls into them, including light, is trapped. But the swift motions of gas and stars near an otherwise invisible object allows astronomers to calculate that it's a black hole and even to estimate its mass.

\section{Space-times}

For physical reasons, a space-time is mathematically defined as a 4-dimensional, smooth, connected, pseudo-Riemannian manifold with a smooth Lorentzian metric of signature $(-,+,+,+)$. By combining space and time into a single manifold, physicists have significantly simplified a large number of physical theories, as well as described in a more uniform way the workings of the universe at both the supergalactic and subatomic levels.

Formerly, from experiments at slow speeds, time was believed to be a constant, which progressed at a fixed rate; however, later high-speed experiments revealed that time slowed down at higher speeds (with such slowing called "time dilation"). Many experiments have confirmed the slowing from time dilation, such as atomic clocks onboard a Space Shuttle running slower than synchronized Earth-bound clocks. Since time varies, it is treated as a variable within the space-time coordinate grid, and time is no longer assumed to be a constant, independent of the location in space.

In order to define Lorentzian space-times, let us recall the definition of pseudoEuclidean spaces. By definition, the pseudo-Euclidean $m$-space $\mathbb{E}_{s}^{m}$ is the Cartesian $m$-space $\mathbf{R}^{m}$ endowed with the canonical pseudo-Euclidean metric of index $s$ given by

$$
g_{0}=-\sum_{i=1}^{s} d x_{i}^{2}+\sum_{j=s+1}^{m} d x_{j}^{2},
$$

where $\left(x_{1}, \ldots, x_{m}\right)$ is a rectangular coordinate system of $\mathbf{R}^{m}$.

The geometry of space-time in special relativity is described by the Minkowski spacetime $\mathbb{E}_{1}^{4}$.

Besides Minkowski space-time, there are two other space-times which are of constant curvature; namely, the de Sitter space-time $S_{1}^{4}$ (or $d S_{4}$ by many physicists) and the anti-de Sitter space-times $H_{1}^{4}$ (or $A d S_{4}$ ).

De Sitter space-time can be defined as a hypersurface of Minkowski 5-space. Take Minkowski space-time $\mathbb{E}_{1}^{5}$ with the standard metric

$$
g=-d t^{2}+\sum_{i=2}^{5} d x_{i}^{2}
$$

the de Sitter space-time $S_{1}^{4}\left(c^{2}\right)$ is the hypersurface described by the hyperboloid

$$
-t^{2}+\sum_{i=2}^{5} x_{i}^{2}=c^{2} .
$$


where $c$ is some positive constant. The metric on de Sitter space-time is the metric induced from the ambient Minkowski metric.

Similarly, an anti de Sitter space-time $H_{1}^{4}\left(-c^{2}\right)$ can be realized as a hypersurface of the pseudo-Euclidean space $\mathbb{E}_{2}^{5}$ with index 2 described by

$$
-t^{2}-x_{2}^{2}+x_{3}^{2}+x_{4}^{2}+x_{5}^{2}=-c^{2} .
$$

where $c$ is some positive constant.

Another important cosmological model in general relativity is the Robertson-Walker space-time described as the warped product:

$$
L_{1}^{4}(f, c):=\left(I \times S, g_{f}^{c}\right), \quad g_{f}^{c}=-d t^{2}+f^{2}(t) g_{c},
$$

where $\left(S, g_{c}\right)$ is a 3-dimensional space of constant curvature $c$. It describes a simplyconnected, homogeneous, isotropic expanding or contracting universe.

Robertson-Walker space-times provide good descriptions of our Universe except in the earliest and the final era (cf. [31]).

A vector $v$ in a space-time (or more generally in a pseudo-Riemannian manifold) is called space-like (respectively, time-like) if $\langle v, v\rangle>0$ (respectively, $\langle v, v\rangle<0$ ). A vector $v$ is called light-like if it is nonzero and it satisfies $\langle v, v\rangle=0$.

A curve in a space-time is called a null curve if its velocity vector is light-like at each point. A surface is called space-like if every nonzero tangent vector of the surface is space-like; and it is called Lorentzian if its metric is Lorentzian (i.e., a metric with index one).

\section{Trapped surfaces}

The concept of trapped surfaces, introduced by Sir Roger Penrose in 1965 (see [32]) plays extremely important role in general relativity and cosmology. It is considered as a cornerstone for the achievement of the singularity theorems, the analysis of gravitational collapse, the cosmic censorship hypothesis, the Penrose inequality, ... etc. (see [10] for a recent survey on marginally trapped surfaces and a closely related subject; namely, the Kaluza-Klein theory).

In the theory of cosmic black holes, if there is a massive source inside the surface, then close enough to a massive enough source, the outgoing light rays may also be converging; a trapped surface. Everything inside is trapped. Nothing can escape, not even light.

It is believed that there will be a marginally trapped surface, separating the trapped surfaces from the untrapped ones, where the outgoing light rays are instantaneously parallel.

In terms of the mean curvature vector field; a space-like surface in a 4-dimensional space-time is marginally trapped if and only if its mean curvature vector field is light-like at each point on the surface. 
The surface of a black hole is the marginally trapped surface. As times develops, the marginally trapped surface generates a hypersurface in space-time, a trapping horizon.

Although many physicists are interested in marginally trapped surfaces, almost no classification results on marginally trapped surfaces are known from differential geometric point of view until the last few years. Moreover, the issue of differentiability of the boundary of the trapped region is still wide open in general relativity theory. However, for strictly stable outer marginally trapped surfaces, the following two results were shown in $[2]$ :

(i) Local existence of a trapping horizon; and

(ii) Outgoing light rays are converging just inside and diverging just outside such a surface.

\section{Marginally trapped surfaces, biharmonicity and light cones}

Let $L: M \rightarrow \mathbb{E}_{s}^{4}$ be an isometric immersion of a pseudo-Riemannian surface $M$ into the pseudo-Euclidean 4 -space $\mathbb{E}_{s}^{4}$ with index $s$. Denote by $\Delta$ the Laplace operator on $M$. Then $L: M \rightarrow \mathbb{E}_{s}^{4}$ is a minimal immersion if and only if $L$ is a harmonic map, i.e., $\Delta L=0$.

An immersion $L: M \rightarrow \mathbb{E}_{s}^{4}$ is called biharmonic if and only if (cf. [5]).

$$
\Delta^{2} L=0
$$

holds identically on $M$.

As far as I know, the first classification result on marginally trapped surfaces from differential geometric viewpoint is related with biharmonic surfaces, which was obtained in [17, Chen-Ishikawa].

Theorem 4.1. Let $L: M \rightarrow \mathbb{E}_{1}^{4}$ be a biharmonic surface in Minkowski space-time $\mathbb{E}_{1}^{4}$ with flat normal connection. Then $L$ is marginally trapped if and only if, up to rigid motions of $\mathbb{E}_{1}^{4}$, the surface is given by

$$
L(u, v)=(\varphi(u, v), u, v, \varphi(u, v))
$$

where $\varphi$ is proper biharmonic function on $M$, i.e., $\Delta \varphi \neq 0$ and $\Delta^{2} \varphi=0$.

The light cone in Minkowski space-time is defined as

$$
\mathcal{L} C_{1}^{4}=\left\{x \in \mathbb{E}_{1}^{4}:\langle x, x\rangle=0\right\} .
$$

Marginally trapped surfaces in light cones are completely determined in $[11,13]$.

Proposition 4.1. Let $x: M \rightarrow \mathcal{L} C_{1}^{3}$ be an isometric immersion of a spatial surface $M$ into the light cone $\mathcal{L} C_{1}^{3} \subset \mathbb{E}_{1}^{4}$. Then $M$ is marginally trapped in $\mathbb{E}_{1}^{4}$ if and only if $M$ is flat. 
Proposition 4.2. Let $M$ be a spatial surface in the de Sitter space-time $S_{1}^{4}(1)$. If $M$ lies in $\mathcal{L C}_{1}=\left\{(y, 1) \in S_{1}^{4}(1) \subset \mathbb{E}_{1}^{5}: y \in \mathbb{E}_{1}^{4},\langle y, y\rangle=0\right\}$, then $M$ is marginally trapped in $S_{1}^{4}(1)$ if and only if it is of curvature one.

Proposition 4.3. Let $M$ be a spatial surface in the anti-de Sitter space-time $H_{1}^{4}(-1)$. If $M$ lies in $\left\{(1, y) \in H_{1}^{4}(-1) \subset \mathbb{E}_{2}^{5}: y \in \mathbb{E}_{2}^{4},\langle y, y\rangle=0\right\}$, then $M$ is marginally trapped if and only if it is of curvature -1 .

\section{Marginally trapped surfaces with positive relative nullity}

Let $M$ be a surface in a space-time $\tilde{M}_{1}^{4}$. Denote by $h$ the second fundamental form of $M$ in $\tilde{M}_{1}^{4}$. The relative null space at a point $p \in M$ in $\tilde{M}_{1}^{4}$ is defined by

$$
\mathcal{N}_{p}(M)=\left\{X \in T_{p} M \mid h(X, Y)=0 \text { for all } Y \in T_{p} M\right\}
$$

The dimension of $\mathcal{N}_{p}(M)$, denoted by $\nu_{p}(M)$, is called the relative nullity at $p$. The surface $M$ is said to have positive relative nullity if $\nu_{p}(M)>0$ for each $p \in M$.

For marginally trapped surfaces with positive nullity in the Minkowski space-time, we have the following classification obtained in [20, Chen-Van der Veken].

Theorem 5.1. Up to Minkowskian motions, there exist two families of marginally trapped surfaces with positive relative nullity in $\mathbb{E}_{1}^{4}$ :

(1) A surface defined by

$$
(f(x), x, y, f(x)),
$$

where $f(x)$ is an arbitrary differentiable function with $f^{\prime \prime}(x)$ being nowhere zero.

(2) A surface defined by

$$
\begin{aligned}
& \left(\int_{0}^{x} r(x) q^{\prime}(x) d x+q(x) y, y \cos x-\int_{0}^{x} r(x) \sin x d x\right. \\
& \left.y \sin x+\int_{0}^{x} r(x) \cos x d x, \int_{0}^{x} r(x) q^{\prime}(x) d x+q(x) y\right),
\end{aligned}
$$

where $q$ and $r$ are defined on an open interval $I \ni 0$ satisfying $q^{\prime \prime}(x)+q(x) \neq 0$ for each $x \in I$.

Conversely, every marginally trapped surface with positive relative nullity in the Minkowski space-time $E_{1}^{4}$ is congruent to an open portion of a surface obtained from the two families.

Chen and Van der Veken also obtained the following complete classification of marginally trapped surfaces with positive relative nullity in de Sitter and anti-de Sitter space-times (cf. [20]). 
Theorem 5.2. Up to rigid motions of $S_{1}^{4}(1)$, there exist two families of marginally trapped surfaces with positive relative nullity in the de Sitter space-time $S_{1}^{4}(1) \subset \mathbb{E}_{1}^{5}$ :

(1) A surface given by

$$
(f(x) \cos y, \sin x \cos y, \sin y, \cos x \cos y, f(x) \cos y),
$$

where $f$ is an arbitrary function defined on an open interval I satisfying $f^{\prime \prime}+f \neq 0$ at each point in $I$.

(2) A surface given by

$$
\begin{aligned}
& \left(p(s), \eta_{1}(s), \eta_{2}(s), \eta_{3}(s), p(s)\right) \cos y \\
& \quad-\left(b-\int_{0}^{s} r(s) p^{\prime}(s) d s, \xi_{1}(s), \xi_{2}(s), \xi_{3}(s), b-\int_{0}^{s} r(s) p^{\prime}(s) d s\right) \sin y
\end{aligned}
$$

where $b$ is a real number, $p$ and $r$ are defined on an open interval $I \ni 0$ such that $r$ is non-constant, $\eta=\left(\eta_{1}, \eta_{2}, \eta_{3}\right)$ is a unit speed curve in $S^{2}(1) \subset \mathbb{E}^{3}$ with geodesic curvature $\kappa_{g}=r$, and $\xi=\left(\xi_{1}, \xi_{2}, \xi_{3}\right)$ is the unit normal of $\eta$ in $S^{2}(1)$.

Conversely, every marginally trapped surface with positive relative nullity in the de Sitter space-time $S_{1}^{4}$ is congruent to an open portion of a surface obtained from the two families.

Theorem 5.3. Up to rigid motions of $H_{1}^{4}(-1)$, there exist five families of marginally trapped surfaces with positive relative nullity in the anti-de Sitter space-time $H_{1}^{4}(-1)$ :

(1) $(f(x) \cosh y, \cosh x \cosh y, \sinh y, \sinh x \cosh y, f(x) \cosh y)$, where $f(x)$ is defined on an open interval $I$ such that $f^{\prime \prime}(x)-f(x) \neq 0$ at each $x \in I$.

(2) $(f(x) \sinh y, \cosh y, \cos x \sinh y, \sin x \sinh y, f(x) \sinh y)$, where $f(x)$ is defined on an open interval $I$ such that $f^{\prime \prime}(x)+f(x) \neq 0$ at each $x \in I$.

(3) $\left(x^{2} e^{y}, \frac{3 e^{y}}{2}-2 \sinh y, e^{y}-2 \sinh y, x e^{y}, x^{2} e^{y}-\frac{e^{y}}{2}\right)$.

(4) $\left(\sinh y-\frac{x^{2} e^{y}}{2}-e^{y}, f(x) e^{y}, x e^{y}, f(x) e^{y}, \sinh y-\frac{x^{2} e^{y}}{2}\right)$, where $f(x)$ is defined on an open interval $I$ such that $f^{\prime \prime}(x) \neq 0$ at each $x \in I$.

(5) A surface defined by

$$
\begin{aligned}
((p(s), & \left.\eta_{1}(s), \eta_{2}(s), \eta_{3}(s), p(s)\right) \cosh y \\
& -\left(b-\int_{0}^{s} r(s) p^{\prime}(s) d s, \xi_{1}(s), \xi_{2}(s), \xi_{3}(s), b-\int_{0}^{s} r(s) p^{\prime}(s) d s\right) \sinh y,
\end{aligned}
$$

where $b$ is a real number, $p$ and $r$ are defined on an open interval $I \ni 0$ such that $r$ is non-constant, $\eta=\left(\eta_{1}, \eta_{2}, \eta_{3}\right)$ is a unit speed curve in $H^{2}(-1) \subset \mathbb{E}_{1}^{3}$ with geodesic curvature $\kappa_{g}=r$, and $\xi=\left(\xi_{1}, \xi_{2}, \xi_{3}\right)$ is the unit normal of $\eta$ in $H^{2}(-1)$. 
Conversely, every marginally trapped surfaces with positive relative nullity in the antide Sitter space-time $H_{1}^{4}$ is congruent to an open portion of a surface obtained from the five families.

\section{Marginally trapped surfaces with $D H=0$}

A surface in a space-time is called parallel if its second fundamental form $h$ is parallel with respect to the Van der Waerden - Borolotti connection, that is, $\bar{\nabla} h=0$ (cf. [4]).

Parallel space-like and Lorentzian surfaces in 4-dimensional Lorentzian space forms were classified by Chen and Van der Veken in [22]. Recently, all parallel space-like surfaces in pseudo-Riemannian space forms with arbitrary index and arbitrary dimension were completely classified by the author in [14].

The following classification of of marginally trapped surfaces with parallel mean curvature vector in Minkowski space-time were obtained from [23, Chen-Van der Veken] together with Proposition 4.1.

Theorem 6.1. Let $M$ be a marginally trapped surface with parallel mean curvature vector in the Minkowski space-time $\mathbb{E}_{1}^{4}$. Then, with respect to suitable Minkowskian coordinates $\left(t, x_{2}, x_{3}, x_{4}\right)$ on $\mathbb{E}_{1}^{4}, M$ is an open part of one of the following six types of surfaces:

(1) a flat parallel biharmonic surface given by

$$
\frac{1}{2}\left((1-b) u^{2}+(1+b) v^{2},(1-b) u^{2}+(1+b) v^{2}, 2 u, 2 v\right), \quad b \in \mathbf{R} .
$$

(2) a flat parallel surface given by

$$
a(\cosh u, \sinh u, \cos v, \sin v), \quad a>0 .
$$

(3) a flat surface given by

$$
(f(u, v), u, v, f(u, v))
$$

where $f$ is a function on $M$ such that $\Delta f=c$ for some nonzero real number $c$.

(4) a flat surface lying in the light cone $\mathcal{L C}$.

(5) a non-parallel surface lying in the de Sitter space-time $S_{1}^{3}\left(r^{2}\right)$ for some $r>0$ such that the mean curvature vector $H^{\prime}$ of $M$ in $S_{1}^{3}\left(r^{2}\right)$ satisfies $\left\langle H^{\prime}, H^{\prime}\right\rangle=-r^{2}$.

(6) a non-parallel surface lying in the hyperbolic space $H^{3}\left(-r^{2}\right)$ for some $r>0$ such that the mean curvature vector $H^{\prime}$ of $M$ in $H^{3}\left(-r^{2}\right)$ satisfies $\left\langle H^{\prime}, H^{\prime}\right\rangle=r^{2}$.

Conversely, all surfaces of types (1)-(6) above give rise to marginally trapped surfaces with parallel mean curvature vector in $\mathbb{E}_{1}^{4}$. 
The following two results from [23] classifies marginally trapped surface with $D H=0$ in de Sitter and anti de Sitter 4-spaces.

Theorem 6.2. Let $M$ be a marginally trapped surface with parallel mean curvature vector in the de Sitter space-time $S_{1}^{4}(1) \subset \mathbb{E}_{1}^{5}$. Then $M$ is congruent to an open part of one of the following eight types of surfaces:

(i) a parallel surface of curvature one given by

$$
(1, \sin u, \cos u \cos v, \cos u \sin v, 1) .
$$

(ii) a flat parallel surface defined by

$$
\frac{1}{2}\left(2 u^{2}-1,2 u^{2}-2,2 u, \sin 2 v, \cos 2 v\right) .
$$

(iii) a flat parallel surface defined by

$$
\left(\frac{b}{\sqrt{4-b^{2}}}, \frac{\cos (\sqrt{2-b} u)}{\sqrt{2-b}}, \frac{\sin (\sqrt{2-b} u)}{\sqrt{2-b}}, \frac{\cos (\sqrt{2+b} v)}{\sqrt{2+b}}, \frac{\sin (\sqrt{2+b} v)}{\sqrt{2+b}}\right)
$$

with $|b|<2$.

(iv) a flat parallel surface defined by

$$
\left(\frac{\cosh (\sqrt{b-2} u)}{\sqrt{b-2}}, \frac{\sinh (\sqrt{b-2} u)}{\sqrt{b-2}}, \frac{\cos (\sqrt{2+b} v)}{\sqrt{2+b}}, \frac{\sin (\sqrt{2+b} v)}{\sqrt{2+b}}, \frac{b}{\sqrt{b^{2}-4}}\right)
$$

with $b>2$.

(v) a surface of constant curvature one immersed in $S_{1}^{4}(1) \subset \mathbb{E}_{1}^{5}$ given by

$$
(f, \cos u, \sin u \cos v, \sin u \sin v, f),
$$

where $f$ is a function satisfies $\Delta f=k$ for some nonzero real number $k$.

(vi) a non-parallel surface of curvature one in $S_{1}^{4}(1)$ which lies in

$$
\mathcal{L C}_{1}:=\left\{(\mathbf{y}, 1) \in \mathbb{E}_{1}^{5}:\langle\mathbf{y}, \mathbf{y}\rangle=0, \mathbf{y} \in \mathbb{E}_{1}^{4}\right\} \subset S_{1}^{4}(1) ;
$$

(vii) a non-parallel surface of $S_{1}^{4}(1)$ which lies in $S_{1}^{4}(1) \cap S_{1}^{4}\left(c_{0}, r^{2}\right)$ with $c_{0} \neq 0$ and $r>0$ such that the mean curvature vector $H^{\prime}$ of $M$ in $S_{1}^{4}(1) \cap S_{1}^{4}\left(c_{0}, r^{2}\right)$ satisfies $\left\langle H^{\prime}, H^{\prime}\right\rangle=1-r^{2}$.

(viii) a non-parallel surface of $S_{1}^{4}(1)$ which lies in $S_{1}^{4}(1) \cap H^{4}\left(c_{0},-r^{2}\right)$ with $c_{0} \neq 0$ and $r>0$ such that the mean curvature vector $H^{\prime}$ of $M$ in $S_{1}^{4}(1) \cap H^{4}\left(c_{0},-r^{2}\right)$ satisfies $\left\langle H^{\prime}, H^{\prime}\right\rangle=1+r^{2}$.

Conversely, all surfaces of types (i)-(viii) above give rise to marginally trapped surfaces with parallel mean curvature vector in $S_{1}^{4}(1)$. 
We also have the following result which classify marginally trapped surfaces with $D H=0$ in $H_{1}^{4}(-1)$.

Theorem 6.3. Let $M$ be a marginally trapped surface with parallel mean curvature vector in the anti de Sitter space-time $H_{1}^{4}(-1) \subset \mathbb{E}_{2}^{5}$. Then, $M$ is congruent to one of the following eight types of surfaces:

(a) a curvature -1 parallel surface given by

$$
(1, \cosh u \cosh v, \sinh u, \cosh u \sinh v, 1) .
$$

(b) a flat parallel surface defined by

$$
\frac{1}{2}\left(2 u^{2}+2, \cosh 2 v, 2 u, \sinh 2 v, 2 u^{2}+1\right) .
$$

(c) a flat parallel surface defined by

$$
\left(\frac{\cosh (\sqrt{2-b} u)}{\sqrt{2-b}}, \frac{\cosh (\sqrt{2+b} v)}{\sqrt{2+b}}, \frac{\sinh (\sqrt{2-b} u)}{\sqrt{2-b}}, \frac{\sinh (\sqrt{2+b} v)}{\sqrt{2+b}}, \frac{b}{\sqrt{4-b^{2}}}\right)
$$

with $|b|<2$.

(d) a flat parallel surface defined by

$$
\left(\frac{b}{\sqrt{b^{2}-4}}, \frac{\cosh (\sqrt{b+2} v)}{\sqrt{b+2}}, \frac{\sinh (\sqrt{b+2} v)}{\sqrt{b+2}}, \frac{\cos (\sqrt{b-2} u)}{\sqrt{b-2}}, \frac{\sin (\sqrt{b-2} u)}{\sqrt{b-2}}\right)
$$

with $b>2$.

(e) a surface of constant curvature -1 immersed in $H_{1}^{4}(-1) \subset \mathbb{E}_{2}^{5}$ given by

$$
(f, \cosh u, \sinh u \cos v, \sinh u \sin v, f),
$$

where $f$ is a function satisfies $\Delta f=k$ for some nonzero real number $k$;

(f) a non-parallel surface of $H_{1}^{4}(-1)$ with curvature -1, lying in

$$
\mathcal{L C}_{2}:=\left\{(1, \mathbf{y}) \in \mathbb{E}_{2}^{5}:\langle\mathbf{y}, \mathbf{y}\rangle=0, \mathbf{y} \in \mathbb{E}_{1}^{4}\right\} \subset H_{1}^{4}(-1) ;
$$

(g) a non-parallel surface lying in $H_{1}^{4}(-1) \cap S_{2}^{4}\left(c_{0}, r^{2}\right)$ with $c_{0} \neq 0$ and $r>0$ such that the mean curvature vector $H^{\prime}$ in $H_{1}^{4}(-1) \cap S_{2}^{4}\left(c_{0}, r^{2}\right)$ satisfies $\left\langle H^{\prime}, H^{\prime}\right\rangle=-r^{2}-1$;

(h) a non-parallel surface lying in $H_{1}^{4}(-1) \cap H_{1}^{4}\left(c_{0},-r^{2}\right)$ with $c_{0} \neq 0$ and $r>0$ such that mean curvature vector $H^{\prime}$ in $H_{1}^{4}(-1) \cap H_{1}^{4}\left(c_{0},-r^{2}\right)$ satisfies $\left\langle H^{\prime}, H^{\prime}\right\rangle=r^{2}-1$.

Conversely, all surfaces of types $(a)-(h)$ above give rise to marginally trapped surfaces with parallel mean curvature vector in $H_{1}^{4}(-1)$. 
Remark 1. Recently, space-like surfaces with $D H=0$ in pseudo-Riemannian space forms with arbitrary index and arbitrary dimension were completely classified by the author in $[11,13]$. In particular, he completely classifies space-like surfaces with $D H=0$ in Lorentzian space forms of arbitrary dimension.

One may also study marginally trapped surfaces in a pseudo-Riemannian $m$-manifold $\tilde{M}_{s}^{m}$ with index $s$. Here, by a marginally trapped surface in $\tilde{M}_{s}^{m}$, we mean a space-like surface whose mean curvature vector field is light-like at each point.

For marginally trapped surfaces in $\mathbb{E}_{s}^{m}$ with $m \geq 5$ we have the following result from [11].

Theorem 6.4. Let $L: M \rightarrow \mathbb{E}_{s}^{m}$ be a marginally trapped surface in a pseudo-Euclidean $m$-space $\mathbb{E}_{s}^{m}$ with index s. If $M$ has parallel mean curvature vector, then $M$ is congruent to one of the following two types of surfaces:

(1) a surface given by

$$
L=(f, \psi, f),
$$

where $f$ is a function on $M$ satisfying $\Delta f=b$ for some real number $b \neq 0$ and $\psi: M \rightarrow \mathbb{E}_{s-1}^{m-2}$ is an isometric minimal immersion;

(2) a marginally trapped surface lying in a totally geodesic Minkowski 4-space $\mathbb{E}_{1}^{4} \subset \mathbb{E}_{s}^{m}$.

\section{Further results on marginally trapped surfaces}

In this section, we present some more results on marginally trapped surfaces.

\subsection{A representation formula}

A conformal representation formula of Weierstrass-Bryant type was obtained in [1, Aledo-Gálvez-Mira] for the class of marginally trapped surfaces $M$ in the Minkowski space-time $\mathbb{E}_{1}^{4}$ which satisfy the following two additional conditions:

(a) $M$ has flat normal connection in $\mathbb{E}_{1}^{4}$, and

(2) $M$ is locally isometric either to a minimal surface in $\mathbb{E}^{3}$ or to a maximal surface in $\mathbb{E}_{1}^{3}$.

\subsection{Marginally trapped surfaces in Robertson-Walker space-times}

For marginally trapped surfaces lying in a Robertson-Walker space-time, we have the following result from [21, Chen-Van der Veken].

Theorem 7.1. Let $L_{1}^{4}(f, c)=I \times_{f} S$ be a Robertson-Walker space-time which contains no open subsets of constant curvature. Then $L_{1}^{4}(f, c)$ does not admit any marginally trapped surface $M$ with positive relative nullity. 
Remark 2. If we do not assume $M$ to have positive relative nullity, there exist marginally trapped surfaces in Robertson-Walker space-times of non-constant sectional curvature.

\subsection{Boost invariant marginally trapped surfaces in $\mathbb{E}_{1}^{4}$}

The boost group in Minkowski space-time $\mathbb{E}_{1}^{4}$ is defined by

$$
G=\left\{\left(\begin{array}{cccc}
\cosh \theta \sinh \theta & 0 & 0 \\
\sinh \theta & \cosh \theta & 0 & 0 \\
0 & 0 & 1 & 0 \\
0 & 0 & 0 & 1
\end{array}\right): \theta \in \mathbf{R}\right\} .
$$

It is known in general relativity theory that the inertial vacuum state of Minkowski space-time is a thermal state when analyzed regarding the notion of time translations defined by a one-parameter family of Lorentz boosts. The orbits of these boost isometries correspond to a family of uniformly accelerating observers. This result, known as the Unruh effect, is actually a consequence of the fact that the Lorentz boost isometries possess a Killing horizon, that is, a null surface which is normal to the Killing field generating the isometries.

Recently, marginally trapped surfaces in $\mathbb{E}_{1}^{4}$ invariant under boost group are obtained by Haesen and Ortega in [28]. In particular, they showed that there exist no $G$-invariant extremal surfaces in Minkowski space-time with constant Gaussian curvature.

\subsection{Marginally trapped surfaces in strictly stationary space-times}

A space-time is called strictly stationary if it contains a Killing vector field which is time-like everywhere.

Mars and Senovilla [30] proved the following non-existence result for strictly stationary space-time.

Theorem 7.2. There do not exist closed marginally trapped surfaces in strictly stationary space-times.

\section{Quasi-minimal flat surfaces in $\mathbb{E}_{2}^{4}$}

There is an important subject closely related with marginally trapped surfaces in space-times; namely, the study of quasi-minimal surfaces. A Lorentzian surface in a pseudo-Riemannian manifold is called quasi-minimal if its mean curvature vector is lightlike at each point (cf. $[33,35])$.

Let us define $a * b$ as

$$
a * b=\left(a_{1} b_{1}, \ldots, a_{4} b_{4}\right)
$$


for any two vectors $a=\left(a_{1}, \ldots, a_{4}\right), b=\left(b_{1}, \ldots, b_{4}\right)$ in $\mathbb{E}_{2}^{4}$.

Flat quasi-minimal surfaces in the pseudo-Euclidean 4 -space $\mathbb{E}_{2}^{4}$ were classified in $[8,25]$.

Theorem 8.1. If $L: M \rightarrow \mathbb{E}_{2}^{4}$ is a quasi-minimal flat surface in $\mathbb{E}_{2}^{4}$, then $L$ is congruent to a surface of the following night types:

(1) A surface defined by

$$
\frac{1}{\sqrt{2}}(\varphi(x, y), x+y, x-y, \varphi(x, y))
$$

where $\varphi(x, y)$ is a function with $\varphi_{x y}>0$ on an open domain $U \subset \mathbb{E}_{1}^{2}$.

(2) A surface defined by

$$
L(x, y)=z(x) y+w(x),
$$

where $z(x)$ is a null curve in the light-cone $\mathcal{L C}$ and $w(x)$ is a null curve satisfying $\left\langle z^{\prime}, w^{\prime}\right\rangle=0$ and $\left\langle z, w^{\prime}\right\rangle=-1$.

(3) A surface defined by

$$
\begin{array}{r}
\frac{1}{2 a b}(2 a b \cos a x \cos b y-\sin a x \sin b y, 2 a b \cos a x \sin b y+\sin a x \cos b y, \\
2 a b \cos a x \cos b y+\sin a x \sin b y, 2 a b \cos a x \sin b y-\sin a x \cos b y),
\end{array}
$$

with $a, b>0$

(4) A surface defined by

$$
\begin{array}{r}
\frac{1}{2 a b}(2 a b \cos a x \cosh b y-\sin a x \sinh b y, 2 a b \cos a x \sinh b y+\sin a x \cosh b y, \\
2 a b \cos a x \cosh b y+\sin a x \sinh b y, 2 a b \cos a x \sinh b y-\sin a x \cosh b y)
\end{array}
$$

for some positive numbers $a, b$.

(5) A surface defined by

$\frac{1}{2 a b}(2 a b \cosh a x \cosh b y-\sinh a x \sinh b y, 2 a b \cosh a x \sinh b y+\sinh a x \cosh b y$,

$2 a b \cosh a x \cosh b y+\sinh a x \sinh b y, 2 a b \cosh a x \sinh b y-\sinh a x \cosh b y)$

for some positive numbers $a, b$.

(6) A surface defined by

$$
L(x, y)=z(y) \cos a x+w(y) \sin a x,
$$

where $a$ is a positive number and $z, w$ are null curves lying in the light cone $\mathcal{L C}$ satisfying $\langle z, w\rangle=z^{\prime \prime}+\delta z=w^{\prime \prime}+\delta w=0$ and $\left\langle z, w^{\prime}\right\rangle=a^{-1}$ for some non-constant function $\delta(y)$. 
(7) A ssurfaces lying in the light cone $\mathcal{L} C \subset \mathbb{E}_{2}^{4}$ given by

$$
L(x, y)=u(x) * z(y)+v(x) * w(y),
$$

where $u, v, z, w$ are curves in $\mathbb{E}_{2}^{4}$ satisfying the following conditions:

$$
\begin{aligned}
& u^{\prime \prime}+\beta u=v^{\prime \prime}+\beta v=0, \\
& z^{\prime \prime}+\delta z=w^{\prime \prime}+\delta w=0, \\
& \left\langle u^{\prime} * z+v^{\prime} * w, u * z^{\prime}+v * w^{\prime}\right\rangle=-1 .
\end{aligned}
$$

where $\beta(x)$ and $\delta(y)$ are non-constant real-valued functions.

(8) A surface defined by

$$
L(x, y)=z(y) \cosh a x+w(y) \sinh a x,
$$

where $a$ is a positive number and $z, w$ are null curves lying in the light cone $\mathcal{L C}$ satisfying $\langle z, w\rangle=z^{\prime \prime}+\delta z=w^{\prime \prime}+\delta w=0$ and $\left\langle z, w^{\prime}\right\rangle=a^{-1}$ for some non-constant function $\delta(y)$.

(9) A surface $\psi_{\gamma \delta}: M \rightarrow \mathbb{E}_{2}^{4}$ defined in Proposition 8.1 given below.

Proposition 8.1. Let $U$ be a simply-connected domain of the Lorentzian plane $\mathbb{E}_{1}^{2}=$ $\{(x, y): x, y \in \mathbf{R}\}$ with metric $g=-d x d y$. Let $M$ denote the flat surface given by $U$ together with the flat metric $g=-d x d y$. Assume that $\gamma$ and $\delta$ are non-constant functions defined on $M$ satisfying

$$
\begin{aligned}
& \gamma_{x}+(\ln \delta)_{x} \gamma=(\ln \beta)_{y}, \quad \delta_{x} \neq 0, \quad(\gamma \delta)_{x} \neq 0, \\
& (\ln \delta)_{x y}-(\ln \beta)_{x y}=2 \beta \gamma
\end{aligned}
$$

with

$$
\beta=\frac{\delta \delta_{x}}{\gamma \gamma_{y}-2 \gamma^{2} \delta_{x}-\gamma_{y} \delta-2 \gamma \gamma_{x} \delta} .
$$

Then, up to rigid motions of the pseudo-Euclidean space $\mathbb{E}_{2}^{4}$, there exists a unique quasiminimal isometric immersion $\psi_{\gamma \delta}: M \rightarrow \mathbb{E}_{2}^{4}$ whose second fundamental form $h$ and normal connection D satisfy

$$
\begin{aligned}
& h\left(\frac{\partial}{\partial x}, \frac{\partial}{\partial x}\right)=\beta e_{4}-\frac{\beta \gamma}{\delta} e_{3}, \quad h\left(\frac{\partial}{\partial x}, \frac{\partial}{\partial y}\right)=e_{3}, \quad h\left(\frac{\partial}{\partial y}, \frac{\partial}{\partial y}\right)=\gamma e_{3}+\delta e_{4}, \\
& D_{\frac{\partial}{\partial x}} e_{3}=\frac{\delta_{x}}{\delta} e_{3}, \quad D_{\frac{\partial}{\partial y}} e_{3}=\frac{\beta_{y}}{\beta} e_{3}, \quad D_{\frac{\partial}{\partial x}} e_{4}=-\frac{\delta_{x}}{\delta} e_{4}, \quad D_{\frac{\partial}{\partial y}} e_{4}=-\frac{\beta_{y}}{\beta} e_{4},
\end{aligned}
$$

for some pseudo-orthonormal normal frame $\left\{e_{3}, e_{4}\right\}$ satisfying

$$
\left\langle e_{3}, e_{3}\right\rangle=\left\langle e_{4}, e_{4}\right\rangle=0, \quad\left\langle e_{3}, e_{4}\right\rangle=-1 \text {. }
$$


As an application of Theorem 8.1 we have the following classification of biharmonic marginally quasi-minimal surfaces in $\mathbb{E}_{2}^{4}$.

Theorem 8.2. A quasi-minimal surface in $\mathbb{E}_{2}^{4}$ is biharmonic if and only if $M$ congruent to one of the following surfaces:

(a) A surface defined by

$$
\frac{1}{\sqrt{2}}(\varphi(x, y), x+y, x-y, \varphi(x, y))
$$

where $\varphi(x, y)$ is a function satisfying $\varphi_{x y} \neq 0$ and $\varphi_{x x y y}=0$ on an open domain $U \subset \mathbb{E}_{1}^{2}$.

(b) A surface defined by

$$
L(x, y)=z(x) y+w(x),
$$

where $z(x)$ is a null curve in the light-cone $\mathcal{L C}$ and $w(x)$ is a null curve satisfying $\left\langle z^{\prime}, w^{\prime}\right\rangle=0$ and $\left\langle z, w^{\prime}\right\rangle=-1$.

\section{Quasi-minimal surfaces with constant positive curvature}

On $\mathbb{E}_{2}^{4}$ we may consider the canonical complex coordinate system $\left\{z_{1}, z_{2}\right\}$ given by $z_{1}=x_{1}+\mathrm{i} x_{2}, z_{2}=x_{3}+\mathrm{i} x_{4}$. The complex structure on $\mathbb{E}_{2}^{4}$ obtained in this way is the standard complex structure on $\mathbb{E}_{2}^{4}$. In this way, $\mathbb{E}_{2}^{4}$ can be regarded as a Lorentzian complex plane $\mathbf{C}_{1}^{2}$.

The following classification of quasi-minimal surfaces of constant positive curvature was obtained in [12].

Theorem 9.1. There exist six families of quasi-minimal surfaces of constant curvature one in $\mathbf{C}_{1}^{2}$ given by the following:

(1) A surface defined by

$$
\left(x+\frac{\mathrm{i} b x^{2}}{\sqrt{2}}+\frac{\sqrt{2} \mathrm{i}-2 b(2 x+y)}{2 b(x+y)^{2}}, x-\frac{\mathrm{i} b x^{2}}{\sqrt{2}}+\frac{\sqrt{2} \mathrm{i}+2 b(2 x+y)}{2 b(x+y)^{2}}\right),
$$

where $b$ is a nonzero real number.

(2) A surface defined by

$$
\begin{aligned}
& \left(\left(\frac{\mathrm{i}-\sqrt{2} b(x+y)}{\sqrt{2} b(x+y)^{2}}+\frac{\sqrt{2} \mathrm{i} b}{a^{2}}\right) \cosh (a x)+\left(\frac{1}{a}-\frac{1}{a(x+y)^{2}}+\frac{\sqrt{2} \mathrm{i} a}{2 b(x+y)}\right) \sinh (a x),\right. \\
& \left.\left(\frac{\mathrm{i}+\sqrt{2} b(x+y)}{\sqrt{2} b(x+y)^{2}}-\frac{\sqrt{2} \mathrm{i} b}{a^{2}}\right) \cosh (a x)+\left(\frac{1}{a}+\frac{1}{a(x+y)^{2}}+\frac{\sqrt{2} \mathrm{i} a}{2 b(x+y)}\right) \sinh (a x)\right),
\end{aligned}
$$

where $a, b$ are nonzero real numbers. 
(3) A surface defined by

$$
\begin{aligned}
& \left(\left(\frac{\mathrm{i}-\sqrt{2} b(x+y)}{\sqrt{2} b(x+y)^{2}}-\frac{\sqrt{2} \mathrm{i} b}{a^{2}}\right) \cos (a x)+\left(\frac{(x+y)^{2}-1}{a(x+y)^{2}}-\frac{\mathrm{i} a}{\sqrt{2} b(x+y)}\right) \sin (a x),\right. \\
& \left.\left(\frac{\mathrm{i}+\sqrt{2} b(x+y)}{\sqrt{2} b(x+y)^{2}}+\frac{\sqrt{2} \mathrm{i} b}{a^{2}}\right) \cos (a x)+\left(\frac{(x+y)^{2}+1}{a(x+y)^{2}}-\frac{\mathrm{i} a}{\sqrt{2} b(x+y)}\right) \sin (a x)\right),
\end{aligned}
$$

where $a, b$ are nonzero real numbers.

(4) A surface defined by

$$
\begin{aligned}
& \frac{\mathrm{i} z(x)+\mathrm{i}(x+y) z^{\prime}(x)}{\sqrt{2} p(x)(x+y)^{2}}-\int^{x}(1+\mathrm{i} k(x)) z(x) d x \\
& \quad+\frac{\mathrm{i}}{\sqrt{2}} \int^{x}\left(\frac{f(x)}{x+y}-\frac{2 p(x)+(x+y) p^{\prime}(x)}{(x+y)^{3} p^{2}(x)}\right) z(x) d x \\
& \quad+\frac{\mathrm{i}}{\sqrt{2}} \int^{y}\left(\int^{x}\left\{\frac{f(x)}{(x+y)^{2}}-\frac{6 p(x)+2(x+y) p^{\prime}(x)}{(x+y)^{4} p^{2}(x)}\right\} z(x) d x\right) d y
\end{aligned}
$$

where $z$ is a null curve lying in $\mathcal{L C}$ satisfying $z^{\prime \prime}(\ln p)^{\prime} z^{\prime}=f z$, and $p, f, k$ are real valued functions with $p \neq 0$.

(5) A surface defined by

$$
z(y)+\frac{\mathrm{i} c_{1}(1+(x+y)(\mathrm{i}+p(y)) q(y))}{(x+y)^{2} q(y)} e^{\int^{y}(\mathrm{i}-p(y)) q(y) d y}
$$

for some real-valued functions $q \neq 0$ and $p$, where $c_{1}$ is a null vector, $z$ is a null curve satisfying $\left\langle c_{1}, z^{\prime}\right\rangle=-2 e^{\int^{y} p q d y} \cos \left(\int^{y} q d y\right)$ and $\left\langle c_{1}, \mathrm{i} z^{\prime}\right\rangle=2 e^{\int^{y} p q d y} \sin \left(\int^{y} q d y\right)$.

(6) A surface defined by

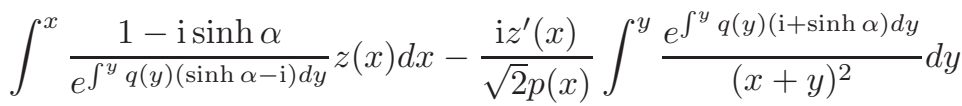

$$
\begin{aligned}
& +\frac{z(x)}{\sqrt{2} \mathrm{i} p(x)} \int^{y} \frac{e^{\int^{y} q(y)(\mathrm{i}+\sinh \alpha) d y}}{(x+y)^{2}}\left(\frac{2}{x+y}-\int^{y} q(y)(\sinh \alpha)_{x} d y\right) d y \\
& -\mathrm{i} \int^{y} \int^{x} \frac{\left(q(y) \cosh \alpha-\alpha_{y}\right) z(x)}{e^{\int^{y} q(y)(\sinh \alpha-\mathrm{i}) d y} \operatorname{sech} \alpha} d x d y,
\end{aligned}
$$

where $\alpha, p, q$ are real-valued functions with $q \neq 0$ and $\alpha_{y} \neq q \cosh \alpha$, and $z$ is a null curve lying in the light cone $\mathcal{L C}$ satisfying $\left\langle z, \mathrm{i} z^{\prime}\right\rangle=2 \sqrt{2} p$.

Conversely, up to rigid motions and dilations, every marginally trapped surface of constant positive curvature in $\mathbf{C}_{1}^{2}$ is locally an open portion of one of the surfaces given by the six families. 


\section{Quasi-minimal surfaces with constant negative curvature}

We also have the following complete classification of quasi-minimal surfaces of constant negative curvature from [12].

Theorem 10.1. There exist six families of quasi-minimal surfaces of constant curvature -1 in $\mathbf{C}_{1}^{2}$ given by the following:

(1) A surface defined by

$$
\begin{aligned}
& \left(\frac{(\mathrm{i}+k)\left(b x^{2}-4 \mathrm{i} x\right)}{4}+\frac{4 \mathrm{i}-2 b x+\sqrt{2} b \sinh (\sqrt{2}(x+y))}{4 b} \operatorname{sech}^{2}\left(\frac{x+y}{\sqrt{2}}\right),\right. \\
& \left.-\frac{(\mathrm{i}+k)\left(b x^{2}+4 \mathrm{i} x\right)}{4}+\frac{4 \mathrm{i}+2 b x-\sqrt{2} b \sinh (\sqrt{2}(x+y))}{4 b} \operatorname{sech}^{2}\left(\frac{x+y}{\sqrt{2}}\right)\right),
\end{aligned}
$$

where $b$ is a nonzero real number and $k$ is an arbitrary real number.

(2) A surface defined by

$$
\begin{aligned}
& \frac{1}{2 a^{2} b}\left(a\left(2 b(1-\mathrm{i} k)-b \operatorname{sech}^{2}\left(\frac{x+y}{\sqrt{2}}\right)-2 \sqrt{2} \mathrm{i} \tanh \left(\frac{x+y}{\sqrt{2}}\right)\right) \sinh (a x)\right. \\
& +\left(b^{2}(\mathrm{i}+k)+2 \mathrm{i} a^{2} \operatorname{sech}^{2}\left(\frac{x+y}{\sqrt{2}}\right)+\sqrt{2} a^{2} b \tanh \left(\frac{x+y}{\sqrt{2}}\right)\right) \cosh (a x) \\
& a\left(b \operatorname{sech}^{2}\left(\frac{x+y}{\sqrt{2}}\right)+2 b(1-\mathrm{i} k)-2 \sqrt{2} \mathrm{i} a^{2}\left(\frac{x+y}{\sqrt{2}}\right)\right) \sinh (a x) \\
& \left.-\left(b^{2}(\mathrm{i}+k)-2 \mathrm{i} a^{2} \operatorname{sech}^{2}\left(\frac{x+y}{\sqrt{2}}\right)+\sqrt{2} a^{2} b \tanh \left(\frac{x+y}{\sqrt{2}}\right)\right) \cosh (a x)\right)
\end{aligned}
$$

where $a, b$ are nonzero real numbers and $k$ is an arbitrary real number.

(3) A surface defined by

$$
\begin{aligned}
& \frac{1}{2 a^{2} b}\left((b \cos (a x)+2 \mathrm{i} a \sin (a x))\left(\sqrt{2} a^{2} \tanh \left(\frac{x+y}{\sqrt{2}}\right)-b(\mathrm{i}+k)\right)\right. \\
& \quad+\frac{2 \mathrm{i} a^{2} \cos (a x)-a b \sin (a x)}{\cosh ^{2}((x+y) / \sqrt{2})}, \frac{2 \mathrm{i} a^{2} \cos (a x)+a b \sin (a x)}{\cosh ^{2}((x+y) / \sqrt{2})} \\
& \left.\quad+(b \cos (a x)-2 \mathrm{i} a \sin (a x))\left(b(\mathrm{i}+k)-\sqrt{2} a^{2} \tanh \left(\frac{x+y}{\sqrt{2}}\right)\right)\right),
\end{aligned}
$$

where $a, b$ are nonzero real numbers and $k$ is an arbitrary real number.

(4) A surface defined by

$$
\begin{aligned}
\int^{x}(1 & -\mathrm{i} \sinh \alpha) z(x) d x+\mathrm{i} \int^{y} \int^{x}(\sinh \alpha)_{y} z(x) d x d y \\
& -\frac{\mathrm{i}}{p(x)}\left(\sqrt{2} \tanh \left(\frac{x+y}{\sqrt{2}}\right) z^{\prime}(x)-\operatorname{sech}^{2}\left(\frac{x+y}{\sqrt{2}}\right) z(x)\right),
\end{aligned}
$$


where $z$ is a null curve in $\mathcal{L C}$ satisfying $z^{\prime \prime}(x)-(\ln p)^{\prime}(x) z^{\prime}(x)=(2+f(x)) z(x)$,

$$
\alpha=\sinh ^{-1}\left(k(x)+\frac{\sqrt{2}(2+f(x))}{p(x) \operatorname{coth}((x+y) / \sqrt{2})}+\frac{p^{\prime}(x)+\sqrt{2} p(x) \tanh ((x+y) / \sqrt{2})}{p^{2}(x) \cosh ^{2}((x+y) / \sqrt{2})}\right),
$$

and $f, p, k$ are real-valued functions with $p \neq 0$.

(5) A surface defined by

$$
z(y)+\frac{\sqrt{2} c_{1}(1-\mathrm{i} p(y))}{e^{\int^{y}(p(y)-\mathrm{i}) q(y) d y}} \tanh \left(\frac{x+y}{\sqrt{2}}\right)+\frac{\mathrm{i} c_{1} \operatorname{sech}^{2}((x+y) / \sqrt{2})}{q(y) e^{\int^{y}(p(y)-\mathrm{i}) q(y) d y}},
$$

where $p, q$ are real-valued functions with $q \neq 0, c_{1}$ is a null vector, and $z$ is a null curve satisfying $\left\langle c_{1}, z^{\prime}\right\rangle=-e^{\int^{y} p q d y} \cos \left(\int^{y} q d y\right)$ and $\left\langle c_{1}, \mathrm{i} z^{\prime}\right\rangle=e^{\int^{y} p q d y} \sin \left(\int^{y} q d y\right)$.

(6) A surface defined by

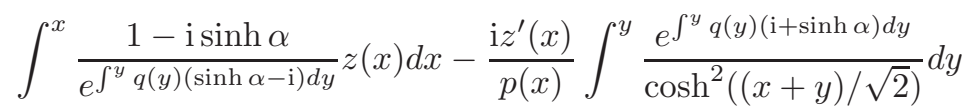

$$
\begin{aligned}
& +\frac{\mathrm{i} z(x)}{p(x)} \int^{y} \frac{e^{\int^{y} q(y)(\mathrm{i}+\sinh \alpha) d y}}{\cosh ^{2}((x+y) / \sqrt{2})}\left(\int^{y} q(y)(\sinh \alpha)_{x} d y-\sqrt{2} \tanh \left(\frac{x+y}{\sqrt{2}}\right)\right) d y \\
& -\mathrm{i} \int^{y} \int^{x} \frac{\left(q(y) \cosh \alpha-\alpha_{y}\right) z(x)}{e^{\int^{y} q(y)(\sinh \alpha-\mathrm{i}) d y} \operatorname{sech} \alpha} d x d y \text {, }
\end{aligned}
$$

where $\alpha, p, q$ are real-valued functions with $q \neq 0$ and $\alpha_{y} \neq q \cosh \alpha$, and $z$ is a null curve lying in the light cone $\mathcal{L C}$ satisfying $\left\langle z, \mathrm{i} z^{\prime}\right\rangle=p$.

Conversely, up to rigid motions and dilations, every marginally trapped surface of constant negative curvature in $\mathbf{C}_{1}^{2}$ is locally an open portion of one of the surfaces given by the six families.

\section{Quasi-minimal surfaces with parallel mean curvature vector in $\mathbb{E}_{2}^{4}$}

Next, we present the classification of quasi-minimal surfaces with parallel mean curvature vector in $\mathbb{E}_{2}^{4}$ obtained in [16, Chen-Garay].

Theorem 11.1. Let $L: M \rightarrow \mathbb{E}_{2}^{4}$ be a flat quasi-minimal surface in $\mathbb{E}_{2}^{4}$. If $M$ has parallel mean curvature vector, then $L$ is congruent to an open portion of one of surfaces of the following seven types:

(1) A surface defined by

$$
\frac{1}{\sqrt{2}}(x y+f(x)+k(y), x+y, x-y, x y+f(x)+k(y)),
$$

for some functions $f(x), k(y)$. 
(2) A surface defined by

$$
L=z(x) y+w(x),
$$

where $z(x)$ is a null curve in the light-cone $\mathcal{L C}$ and $w(x)$ is a null curve satisfying $\left\langle z^{\prime}, w^{\prime}\right\rangle=0,\left\langle z, w^{\prime}\right\rangle=-1$ and $z^{\prime \prime}(x)+\beta(x) z(x)=0$ for some function $\beta(x)$.

(3) A surface defined by

$\frac{1}{2 a b}(2 a b \cos a x \cos b y-\sin a x \sin b y, 2 a b \cos a x \sin b y+\sin a x \cos b y$,

$2 a b \cos a x \cos b y+\sin a x \sin b y, 2 a b \cos a x \sin b y-\sin a x \cos b y), \quad a, b>0$.

(4) A surface defined by

$\frac{1}{2 a b}(2 a b \cos a x \cosh b y-\sin a x \sinh b y, 2 a b \cos a x \sinh b y+\sin a x \cosh b y$,

$2 a b \cos a x \cosh b y+\sin a x \sinh b y, 2 a b \cos a x \sinh b y-\sin a x \cosh b y), \quad a, b>0$.

(5) A surface defined by

$\frac{1}{2 a b}(2 a b \cosh a x \cosh b y-\sinh a x \sinh b y$,

$2 a b \cosh a x \sinh b y+\sinh a x \cosh b y, 2 a b \cosh a x \cosh b y+\sinh a x \sinh b y$,

$2 a b \cosh a x \sinh b y-\sinh a x \cosh b y), \quad a, b>0$.

(6) A surface defined by

$$
L=z(y) \cos a x+w(y) \sin a x,
$$

where $z$ and $w$ are null curves lying in the light cone $\mathcal{L C}$ satisfying $\langle z, w\rangle=z^{\prime \prime}+\delta z=$ $w^{\prime \prime}+\delta w=0$ and $\left\langle z, w^{\prime}\right\rangle=a^{-1}$ for some non-constant function $\delta(y)$ and positive number $a$.

(7) A surface defined by

$$
L=z(y) \cosh a x+w(y) \sinh a x,
$$

where $z$ and $w$ are null curves lying in the light cone $\mathcal{L C}$ satisfying $\langle z, w\rangle=z^{\prime \prime}+\delta z=$ $w^{\prime \prime}+\delta w=0$ and $\left\langle z, w^{\prime}\right\rangle=a^{-1}$ for some non-constant function $\delta(y)$ and positive number $a$.

Theorem 11.2. Let $L: M \rightarrow \mathbf{C}_{1}^{2}$ be a quasi-minimal immersion of a surface into $\mathbf{C}_{1}^{2}$. Assume that $M$ contains no flat points. Then $M$ has parallel mean curvature vector if and only if the immersion $L$ is congruent to $L_{k \psi c}: M_{\psi} \rightarrow \mathbf{C}_{1}^{2}$ as described in Proposition 11.1, where $c$ is a nonzero real number, $k(y)$ a nonzero real-valued function, and $\psi(x, y)$ a real-valued function satisfying $\psi_{x} \neq 0$ and the Riccati equation $\psi_{y}=\epsilon k^{2}(y)\left(\psi^{2}+1\right)-1$ $(\epsilon=1$ or -1$)$. 
Proposition 11.1. Let $c$ be a nonzero real number, $k(y)$ a nonzero real-valued function and $\psi(x, y)$ a real-valued function satisfying $\psi_{x} \neq 0$ and the Riccati equation:

$$
\psi_{y}=\epsilon k^{2}(y)\left(\psi^{2}+1\right)-1, \quad(\epsilon=1 \text { or }-1),
$$

on a simply-connected domain $U$. Let $M_{\psi}=\left(U, g_{\psi}\right)$ be the surface equipped with the Lorentzian metric:

$$
g_{\psi}=-\frac{d x \otimes d y+d y \otimes d x}{\left|\psi_{x}\right|} .
$$

Then the Gaussian curvature of $M_{\psi}$ is $-2 \epsilon \operatorname{sign}\left(\psi_{x}\right) k^{2}(y) \psi_{x}^{2}$. Moreover, up to rigid motions, there exists a unique quasi-minimal immersion $\hat{L}=L_{k \psi c}: M_{\psi} \rightarrow \mathbf{C}_{1}^{2}$ with parallel mean curvature vector and with Wirtinger angle $\alpha=\sinh ^{-1} \psi$ such that the second fundamental form satisfies

$$
\begin{aligned}
& h\left(\frac{\partial}{\partial x}, \frac{\partial}{\partial x}\right)=\alpha_{x}(\tanh \alpha-\mathrm{i} \operatorname{sech} \alpha) \hat{L}_{x}+c(\mathrm{i}+\psi) \hat{L}_{y} \\
& h\left(\frac{\partial}{\partial x}, \frac{\partial}{\partial y}\right)=\operatorname{sech} \alpha(\mathrm{i} \operatorname{sech} \alpha-\tanh \alpha) \hat{L}_{x} \\
& h\left(\frac{\partial}{\partial y}, \frac{\partial}{\partial y}\right)=\frac{\epsilon \alpha_{x} k^{2}(y)}{c}(\tanh \alpha-\mathrm{i} \operatorname{sech} \alpha) \hat{L}_{x}+\epsilon k^{2}(y)(\mathrm{i}+\psi) \hat{L}_{y} .
\end{aligned}
$$

\section{Lorentzian complex space forms and Legendre curves}

\subsection{Lorentzian complex space forms}

Let $\tilde{M}_{i}^{n}(4 \varepsilon)$ be an indefinite complex space form of complex dimension $n$ and complex index $i$. The complex index is defined as the complex dimension of the largest complex negative definite subspace of the tangent space. If $i=1$, we say that $\tilde{M}_{i}^{n}(4 \varepsilon)$ is Lorentzian.

The curvature tensor $\tilde{R}$ of $\tilde{M}_{i}^{n}(4 \varepsilon)$ is given by

$$
\tilde{R}(X, Y) Z=\varepsilon\{\langle Y, Z\rangle X-\langle X, Z\rangle Y+\langle J Y, Z\rangle J X-\langle J X, Z\rangle J Y+2\langle X, J Y\rangle J Z\} .
$$

Let $\mathbf{C}^{n}$ denote the complex number $n$-space with complex coordinates $z_{1}, \ldots, z_{n}$. The $\mathbf{C}^{n}$ endowed with $g_{i, n}$, i.e., the real part of the Hermitian form

$$
b_{i, n}(z, w)=-\sum_{k=1}^{i} \bar{z}_{k} w_{k}+\sum_{j=i+1}^{n} \bar{z}_{j} w_{j}, \quad z, w \in \mathbf{C}^{n}
$$

defines a flat indefinite complex space form with complex index $i$. We simply denote the pair $\left(\mathbf{C}^{n}, g_{i, n}\right)$ by $\mathbf{C}_{i}^{n}$. Consider the differentiable manifold:

$$
S_{2}^{2 n+1}=\left\{z \in \mathbf{C}_{1}^{n+1} ; b_{1, n+1}(z, z)=1\right\},
$$


which is an indefinite real space form of constant sectional curvature one. The Hopf fibration

$$
\pi: S_{2}^{2 n+1} \rightarrow C P_{1}^{n}(4): z \mapsto z \cdot \mathbf{C}^{*}
$$

is a submersion and there exists a unique pseudo-Riemannian metric of complex index one on $C P_{1}^{n}(4)$ such that $\pi$ is a Riemannian submersion. The pseudo-Riemannian manifold $C P_{1}^{n}(4)$ is a Lorentzian complex space form of positive holomorphic sectional curvature 4.

Analogously, consider $H_{3}^{2 n+1}=\left\{z \in \mathbf{C}_{2}^{n+1} ; b_{2, n+1}(z, z)=-1\right\}$, which is an indefinite real space form of constant sectional curvature -1 . The Hopf fibration

$$
\pi: H_{3}^{2 n+1}(-1) \rightarrow C H_{1}^{n}(-4): z \mapsto z \cdot \mathbf{C}^{*}
$$

is also a submersion and there is a unique pseudo-Riemannian metric of complex index 1 on $C H_{1}^{n}(-4)$ such that $\pi$ is a Riemannian submersion. The $C H_{1}^{n}(-4)$ is a Lorentzian complex space form of negative holomorphic sectional curvature -4 .

\subsection{Legendre curves}

Let $\langle$,$\rangle denote the inner product on \mathbf{C}_{i}^{3}$ induced from $g_{i, 3}$ for $i=1$ or 2 . Let us put $\epsilon_{v}=1$ (respectively, $\epsilon_{v}=-1$ ) whenever $v$ is a spacelike vector (respectively, $v$ is timelike).

A curve $z$ in $S_{2}^{5}$, (in $H_{3}^{5}$, or in $\mathcal{L} C$ ) is called Legendre if $\left\langle z^{\prime}, i z\right\rangle=0$ holds identically. The squared Legendre curvature $\hat{\kappa}^{2}$ of a unit speed Legendre curve $z$ is defined by $\hat{\kappa}^{2}=$ $\epsilon_{z^{\prime}}\left\langle z^{\prime \prime}, z^{\prime \prime}\right\rangle$; its Legendre torsion is $\hat{\tau}=\epsilon_{z^{\prime}}\left\langle z^{\prime \prime}, i z^{\prime \prime \prime}\right\rangle$.

If $z(s)$ is a unit speed Legendre curve in $S_{2}^{5}(1) \subset \mathbf{C}_{1}^{3}$ (or in $H_{3}^{5}(-1) \subset \mathbf{C}_{2}^{3}$ ), then $z /|z|, i z /|z|, z^{\prime}, i z^{\prime}$ are orthonormal vector fields defined along the curve. Thus, there exists a unit normal vector field $P_{z}$ such that $z /|z|, i z /|z|, z^{\prime}, i z^{\prime}, P_{z}, i P_{z}$ form an orthonormal frame. By differentiating $\left\langle z^{\prime}(s), i z(s)\right\rangle=0,\left\langle z^{\prime}(s), z(s)\right\rangle=0$, we get $\left\langle z^{\prime \prime}, i z\right\rangle=0$, $\left\langle z^{\prime \prime}, z\right\rangle=-\epsilon_{z^{\prime}}$, Thus, $z^{\prime \prime}$ can be expressed as

$$
z^{\prime \prime}(s)=i \psi(s) z^{\prime}(s)-\epsilon_{z^{\prime}} c z(s)-a(s) P_{z}(s)+b(s) i P_{z}(s)
$$

for some real-valued functions $\psi, a, b$. The Legendre curve $z(s)$ is called special Legendre if (12.1) reduces to

$$
z^{\prime \prime}(s)=i \psi(s) z^{\prime}(s)-\epsilon_{z^{\prime}} c z(s)-a(s) P_{z}(s),
$$

where $P_{z}$ is a unit parallel normal vector field, i.e., $P_{z}^{\prime}(s)=\mu(s) z^{\prime}(s)$ for $\mu=a \epsilon_{z^{\prime}} \epsilon_{P_{z}}$.

A Legendre curve $z$ in the light cone $\mathcal{L} C$ is called special Legendre if $\left\langle i z^{\prime}, z^{\prime \prime}\right\rangle=0$ holds identically.

\section{Quasi-minimal Lagrangian surfaces}

An $n$-dimensional submanifold $M$ of a Kähler (or more generally, of a pseudo-Kähler) $n$-manifold is called Lagrangian if the almost complex structure $J$ of the ambient manifold 
interchanges the tangent and the normal spaces of $M$. Clearly, Lagrangian surfaces of a Lorentzian Kähler surface $\tilde{M}_{1}^{2}$ are always Lorentzian.

Lagrangian submanifolds appear naturally in the context of classical mechanics and mathematical physics. For instance, the systems of partial differential equations of Hamilton 帒Jacobi type lead to the study of Lagrangian submanifolds and foliations in the cotangent bundle. Furthermore, Lagrangian submanifolds are part of a growing list of mathematically rich special geometries that occur naturally in string theory.

Quasi-minimal Lagrangian surfaces in a Lorentzian complex space form whose mean curvature vector $H$ satisfies $\Delta H=\lambda H$ for some constant $\lambda$ were studied by Sasahara in [34].

Recently, quasi-minimal Lagrangian surfaces in Lorentzian complex space forms have been completely classified by Chen and Dillen in [15].

Theorem 13.1. Let $M$ be a marginally trapped Lagrangian surface in the Lorentzian complex plane $\mathbf{C}_{1}^{2}$.

(i) If $M$ is flat, either $M$ is an open part of a Lagrangian surfaces defined by

(i.1) $L(x, y)=e^{i y} z(x)$ for some null curve $z(x)$ in the light cone $\mathcal{L C}$; or $M$ is an open part of a Lagrangian surfaces defined by

(i.2) $L(s, t)=c_{1} s e^{i f(t)}+z(t)$, where $f(t)$ is a real-valued function, $c_{1}$ is a lightlike vector, and $z(t)$ is a null curve in $\mathbf{C}_{1}^{2}$ satisfying $\left\langle i z^{\prime}, e^{i f} c_{1}\right\rangle=0$ and $\left\langle z^{\prime}, e^{i f} c_{1}\right\rangle=-1$.

(ii) If $M$ is not flat, then it is an open part of a Lagrangian surface defined by

$$
L(x, y)=z(x) \int_{y_{0}}^{y} e^{i y} \psi_{x} d y-z^{\prime}(x) \int_{y_{0}}^{y} e^{i y} \psi d y
$$

where $\psi(x, y)$ with $\psi_{y} \neq 0$ is a solution of $\psi_{x x}=q(x) \psi+1$ for some function $q(x)$ and $z(x)$ is curve in the light cone $\mathcal{L C}$ of $\mathbf{C}_{1}^{2}$ satisfying $z^{\prime \prime}=q z$.

Theorem 13.2. Let $M$ be a marginally trapped Lagrangian surface in the Lorentzian complex projective plane $C P_{1}^{2}(4)$.

(p) If $M$ is of constant curvature $K$, then either $K=1$ or $K=0$.

(p.1) If $K=1$, then $M$ is an open part of one of the following four types of Lagrangian surfaces:

(p.1.1) A Lagrangian surface defined by

$$
\frac{1}{a(u+v)}\left(e^{\sqrt{2} i a v}(\sqrt{2}+i a(u-v)), e^{\sqrt{2} i a v} a(u-v), \sqrt{2}+i a(u+v)\right),
$$

where $a$ is a nonzero real number. 
(p.1.2) A Lagrangian surface defined by

$$
\left(\frac{2}{u+v}+\sqrt{2} i f(v)\right) z(v)-z^{\prime}(v)
$$

where $f(v)$ is a non-constant real-valued function and $z(v)$ is a unit speed spacelike Legendre curve with Legendre squared curvature $\hat{\kappa}^{2}=$ $6 f^{2}(v)$ in the light cone $\mathcal{L C}$.

(p.1.3) A Lagrangian surface defined by

$$
\begin{gathered}
\frac{e^{\frac{i}{\sqrt{2}} b v}}{3 b w}\left((\sqrt{6}+i \sqrt{3} b w) \cosh \left(\frac{\sqrt{6}}{2} b v\right)-3(\sqrt{2} i+b w) \sinh \left(\frac{\sqrt{6}}{2} b v\right),\right. \\
\left.\cosh \left(\frac{\sqrt{6}}{2} b v\right)+\sqrt{3}(i b w-2 \sqrt{2}) \sinh \left(\frac{\sqrt{6}}{2} b v\right), \frac{\sqrt{6}+i \sqrt{3} b w}{e^{3 i b v / \sqrt{2}}}\right),
\end{gathered}
$$

where $b$ is a positive real number.

(p.1.4) A Lagrangian surface defined by $L(u, v)=\frac{z(v)}{u+v}-\frac{z^{\prime}(v)}{2}$, where $z(v)$ is a unit speed timelike special Legendre curve in the light cone $\mathcal{L C}$ with zero Legendre curvature $\hat{\kappa}$ and nonzero Legendre torsion $\hat{\tau}$.

(p.2) If $K=0$, it is an open part of one of the following three types of flat Lagrangian surfaces:

(p.2.1) A Lagrangian surface defined by

$$
\frac{e^{2^{-\frac{1}{3}} i x+\frac{1}{3} i y}}{6 \sqrt{2}}\left(3\left(2^{\frac{2}{3}}\right) x+4 y, 8 i+3\left(2^{\frac{2}{3}}\right) x+4 y, 2 \sqrt{2} e^{i y-3\left(2^{-\frac{1}{3}}\right) i x}\right) .
$$

(p.2.2) A Lagrangian surface defined by

$$
\begin{aligned}
& e^{i q x+i b\left(q^{2}-p^{2}\right) y} \\
& \quad \times\left(\frac{i\left(p^{2}-3 q^{2}\right) \cosh (p x+2 b p q y)+4 p q \sinh (p x+2 b p q y)}{p \sqrt{2\left(p^{2}+9 q^{2}\right)}},\right. \\
& \left.\quad \frac{\sqrt{p^{2}+q^{2}}}{\sqrt{p^{2}+9 q^{2}}} e^{i b\left(p^{2}+3 q^{2}\right) y-3 i q x}, \frac{\sqrt{p^{2}+q^{2}}}{\sqrt{2} p} \cosh (p x+2 b p q y)\right),
\end{aligned}
$$

where $b$ is a real number either negative or greater than $\sqrt[3]{4} / 3$ and

$$
p=\frac{2^{-\frac{2}{3}} \sqrt{3} \eta^{2}-3^{\frac{5}{6}} b}{6^{\frac{2}{3}} b \eta}, q=\frac{3^{\frac{1}{3}} b+2^{-\frac{2}{3}} \eta^{2}}{6^{\frac{2}{3}} b \eta}, \eta=\left(9 b^{3}+\sqrt{81 b^{6}-12 b^{3}}\right)^{\frac{1}{3}} .
$$


(p.2.3) A Lagrangian surface defined by

$$
\begin{aligned}
& \left(\frac{\left(4 k r^{2}-\sqrt{3} r\left(k^{2}+r^{2}\right)\right)^{\frac{1}{2}}}{\sqrt{3 k^{2}-9 k r^{2}}} e^{-i(\sqrt{3} k+r) x+i b\left(3 k^{2}+2 \sqrt{3} k r+r^{2}\right) y},\right. \\
& \frac{\left(r^{2}-3 k^{2}\right) e^{2 i r(x+2 b r y)}}{\sqrt{9 k^{4}-30 k^{2} r^{2}+9 r^{4}}}, \frac{\sqrt{3 k^{2} r+2 \sqrt{3} k r^{2}+r^{3}}}{\sqrt{3 k\left(r^{2}-k^{2}\right)+2 \sqrt{3} k^{2} r}} \\
& \left.\quad \times e^{i\left(3 b k^{2} y+\sqrt{3} k(x-2 b r y)-r x+b r^{2} y\right)}\right)
\end{aligned}
$$

where

$$
k=\frac{\left(4 \delta+2^{\frac{2}{3}} \delta^{2}+2^{\frac{4}{3}}\right)^{\frac{1}{2}}}{2 \sqrt{6 b \delta}}, \quad r=\frac{\left(4 \delta-2^{\frac{2}{3}} \delta^{2}-2^{\frac{4}{3}}\right)^{\frac{1}{2}}}{2 \sqrt{6 b \delta}}
$$

and

$$
\delta=\left(2-27 b^{3}+3 \sqrt{81 b^{6}-12 b^{3}}\right)^{\frac{1}{3}}
$$

for some $b \in\left(0, \frac{\sqrt[3]{4}}{3}\right)$.

(q) If $M$ has non-constant curvature, then it is an open part of the Lagrangian surface $\hat{\psi}: M_{\psi} \rightarrow C P_{1}^{2}(4)$ defined in Proposition 13.1 .

Theorem 13.3. Let $M$ be a marginally trapped Lagrangian surface in the Lorentzian complex hyperbolic plane $\mathrm{CH}_{1}^{2}(-4)$.

(h) If $M$ is of constant curvature $K$, then $K=-1$ or $K=0$.

(h.1) If $K=-1$, then $M$ is an open part of one of the following four types of Lagrangian surfaces:

(h.1.1) A Lagrangian surface defined by

$\frac{1}{a(u-v)}\left(a e^{\sqrt{2} i a v}(u+v), a(u-v)+i \sqrt{2}, e^{\sqrt{2} i a v}(a(u+v))+\sqrt{2} i\right)$,

where $a$ is a nonzero real number.

(h.1.2) A Lagrangian surface defined by

$$
\left(\frac{2}{u-v}-\sqrt{2} i \psi(v)\right) z(v)+z^{\prime}(v)
$$

where $\psi(v)$ is a non-constant real-valued function and $z(v)$ is a unit speed timelike Legendre curve with non-constant Legendre squared curvature in the light cone $\mathcal{L C}$. 
(h.1.3) A Lagrangian surface defined by

$$
\begin{aligned}
& \frac{e^{-\frac{i}{\sqrt{2}} b v}}{3 b w}\left(3(b w+i \sqrt{2}) \cosh \left(\frac{\sqrt{6}}{2} b v\right)+3(\sqrt{2}+i b w) \sinh \left(\frac{\sqrt{6}}{2} b v\right)\right. \\
& \quad e^{\frac{3}{\sqrt{2}} i b v}(\sqrt{6}+i \sqrt{3} b w), 3 b w \sinh \left(\frac{\sqrt{6}}{2} b v\right) \\
& \left.\quad-\sqrt{3}(i b w-2 \sqrt{2}) \cosh \left(\frac{\sqrt{6}}{2} b v\right)\right)
\end{aligned}
$$

where $b$ is a nonzero real number.

(h.1.4) A Lagrangian surface defined by $L(u, v)=\frac{z(v)}{u-v}-\frac{z^{\prime}(v)}{2}$, where $z(v)$ is a unit speed spacelike special Legendre curve in the light cone $\mathcal{L C}$ with zero Legendre curvature $\hat{\kappa}$ and nonzero Legendre torsion $\hat{\tau}$.

(h.2) If $K=0$, then $M$ is an open part of one of the following three types of flat Lagrangian surfaces:

(h.2.1) A Lagrangian surface defined by

$$
\frac{e^{\frac{i}{6}\left(2 y-3\left(2^{2 / 3}\right) x\right)}}{3\left(2^{5 / 6}\right)}\left(2^{\frac{5}{6}} e^{i y+3 i x / 2^{\frac{1}{3}}}, 3 i x+2^{\frac{4}{3}}(2-i y), 3 x-2^{\frac{4}{3}} y\right) .
$$

(h.2.2) A flat Lagrangian surface defined by

$$
\begin{aligned}
& e^{i q x+i b\left(q^{2}-p^{2}\right) y}\left(\frac{4 p q \cosh (p x+2 b p q y)+i\left(p^{2}-3 q^{2}\right) \sinh (p x+2 b p q y)}{p \sqrt{2 p^{2}+18 q^{2}}},\right. \\
& \left.\frac{\sqrt{p^{2}+q^{2}}}{\sqrt{p^{2}+9 q^{2}}} e^{i\left(b p^{2} y+3 b q^{2} y-3 q x\right)}, \frac{\sqrt{p^{2}+q^{2}}}{\sqrt{2} p} \sinh (p x+2 b p q y)\right),
\end{aligned}
$$

where $b$ is either a negative number or a positive number greater than $\sqrt[3]{4} / 3$ and

$$
p=\frac{2^{-\frac{2}{3}} \sqrt{3} \eta^{2}-3^{\frac{5}{6}} b}{6^{\frac{2}{3}} b \eta}, q=\frac{2^{-\frac{2}{3}} \eta^{2}+3^{\frac{1}{3}} b}{6^{\frac{2}{3}} b \eta}, \eta=\left(\sqrt{81 b^{6}-12 b^{3}}-9 b^{3}\right)^{\frac{1}{3}} .
$$

(h.2.3) A flat Lagrangian surface defined by

$$
\begin{aligned}
& \left(\frac{\sqrt{3 k^{2} r-2 \sqrt{3} k r^{2}+r^{3}}}{\sqrt{3 \sqrt{3} k\left(k^{2}-r^{2}\right)+6 k^{2} r}} e^{\frac{i}{b}\left(r x+r^{2} y+3 k^{2} y+\sqrt{3} k(x+2 r y)\right)},\right. \\
& \quad \frac{\left(r^{2}-3 k^{2}\right) e^{2 i b^{-1} r(2 r y-x)}}{\sqrt{9 k^{4}-30 k^{2} r^{2}+9 r^{4}}}, \\
& \left.\quad \frac{\sqrt{4 k r^{2}+\sqrt{3} r\left(r^{2}+r^{2}\right)}}{\sqrt{3 k\left(k^{2}-3 r^{2}\right)}} e^{\frac{i}{b}\left(r x+r^{2} y+3 k^{2} y-\sqrt{3} k(x+2 r y)\right)}\right),
\end{aligned}
$$


where $b \in(0, \sqrt[3]{4} / 3)$, and

$$
\begin{aligned}
& k=\frac{\sqrt{b(\gamma+1)^{2}}}{2 \sqrt{3 \gamma}, \quad r=\frac{\sqrt{b(\gamma-1)^{2}}}{2 \sqrt{-3 \gamma}}} \\
& \gamma=\frac{-\sqrt[3]{27 b^{3}-2+3 \sqrt{3} b \sqrt{27 b^{4}-4 b}}}{\sqrt[3]{2}} .
\end{aligned}
$$

(g) If $M$ has non-constant curvature, then it is an open part of the Lagrangian surface $\hat{\phi}: \tilde{P}_{\phi} \rightarrow C H_{1}^{2}(-4)$ defined in Proposition 13.2.

Proposition 13.1. Let $\psi(x, y)$ be a solution of the third order differential equation:

$$
\psi \psi_{x x y}-\psi_{x x} \psi_{y}-3 \psi^{2} \psi_{x}+\psi_{y}=0 .
$$

with $\psi_{x}, \psi_{y} \neq 0$ and let $D_{\psi}$ be a simply-connected domain in $\mathbf{R}^{2}$ such that $\psi$ is nowhere zero on $D_{\psi}$. Denote the surface $\left(D_{\psi}, g_{\psi}\right)$ endowed with Lorentzian metric $g_{\psi}=-\psi d x d y$ by $M_{\psi}$. Then, up to rigid motions of $C P_{1}^{2}(4)$, there exists a unique Lagrangian isometric immersion $\hat{\psi}: M_{\psi} \rightarrow C P_{1}^{2}(4)$ whose second fundamental form satisfies

$$
\begin{aligned}
& h\left(\frac{\partial}{\partial x}, \frac{\partial}{\partial x}\right)=\frac{1}{\psi} J \frac{\partial}{\partial y}, \quad h\left(\frac{\partial}{\partial x}, \frac{\partial}{\partial y}\right)=J \frac{\partial}{\partial x}, \\
& h\left(\frac{\partial}{\partial y}, \frac{\partial}{\partial y}\right)=\left(\psi_{x y}-\frac{\psi_{x} \psi_{y}}{\psi}-\psi^{2}\right) J \frac{\partial}{\partial x}+J \frac{\partial}{\partial y} .
\end{aligned}
$$

Similarly, we have the following.

Proposition 13.2. Let $\phi(x, y)$ be a solution of the third order differential equation:

$$
\phi \phi_{x x y}-\phi_{x x} \phi_{y}+3 \phi^{2} \phi_{x}+\phi_{y}=0 .
$$

with $\phi_{x}, \phi_{y} \neq 0$ and let $D_{\phi}$ be a simply-connected domain in $\mathbf{R}^{2}$ such that $\phi$ is nowhere zero on $D_{\phi}$. Denote the surface $\left(D_{\phi}, g_{\phi}\right)$ endowed with Lorentzian metric $g_{\phi}=-\phi d x d y$ by $P_{\phi}$. Then, up to rigid motions of $C_{1}^{2}(-4)$, there exists a unique Lagrangian isometric immersion $\hat{\phi}: P_{\phi} \rightarrow C H_{1}^{2}(-4)$ whose second fundamental form satisfies

$$
\begin{aligned}
& h\left(\frac{\partial}{\partial x}, \frac{\partial}{\partial x}\right)=\frac{1}{\phi} J \frac{\partial}{\partial x}, \quad h\left(\frac{\partial}{\partial x}, \frac{\partial}{\partial y}\right)=J \frac{\partial}{\partial x}, \\
& h\left(\frac{\partial}{\partial y}, \frac{\partial}{\partial y}\right)=\left(\phi_{x y}-\frac{\phi_{x} \phi_{y}}{\phi}+\phi^{2}\right) J \frac{\partial}{\partial x}+J \frac{\partial}{\partial y} .
\end{aligned}
$$

\section{Slant quasi-minimal surfaces in Lorentzian complex space forms}

First recall the following fundamental result on Lorentzian surface in Lorentzian Kähler surface from [9]. 
Proposition 14.1. Every Lorentzian surface in a Lorentzian Kähler surface contains no complex points.

Let $M$ be a Lorentzian surface in a Lorentzian Kähler surface $\left(\tilde{M}_{1}^{2}, g, J\right)$. For each tangent vector $X$ of $M$, we put

$$
J X=P X+F X
$$

where $P X$ and $F X$ are the tangential and the normal components of $J X$

On $M$ there exists a pseudo-orthonormal local frame $\left\{e_{1}, e_{2}\right\}$ on $M$ such that

$$
\left\langle e_{1}, e_{1}\right\rangle=\left\langle e_{2}, e_{2}\right\rangle=0, \quad\left\langle e_{1}, e_{2}\right\rangle=-1 .
$$

For a pseudo-orthonormal frame $\left\{e_{1}, e_{2}\right\}$ on a Lorentzian surface $M$ satisfying (14.2), it follows from (14.1), (14.2), and $\langle J X, J Y\rangle=\langle X, Y\rangle$ that

$$
P e_{1}=(\sinh \alpha) e_{1}, \quad P e_{2}=-(\sinh \alpha) e_{2}
$$

for some function $\alpha$. If we put

$$
e_{3}=(\operatorname{sech} \alpha) F e_{1}, \quad e_{4}=(\operatorname{sech} \theta) F e_{2},
$$

then we obtain from $(14.1)-(14.4)$ that

$$
\begin{aligned}
& J e_{1}=\sinh \alpha e_{1}+\cosh \alpha e_{3}, \quad J e_{2}=-\sinh \alpha e_{2}+\cosh \alpha e_{4}, \\
& J e_{3}=-\cosh \alpha e_{1}-\sinh \alpha e_{3}, \quad J e_{4}=-\cosh \alpha e_{2}+\sinh \alpha e_{4}, \\
& \left\langle e_{3}, e_{3}\right\rangle=\left\langle e_{4}, e_{4}\right\rangle=0, \quad\left\langle e_{3}, e_{4}\right\rangle=-1,
\end{aligned}
$$

We call such a frame $\left\{e_{1}, e_{2}, e_{3}, e_{4}\right\}$ an adapted pseudo-orthonormal frame for the Lorentzian surface $M$ in $\tilde{M}_{1}^{2}$.

A Lorentzian surface $M$ in a Lorentzian Kähler surface is called $\theta$-slant if the function $\alpha$ defined by $(14.3)$ is a constant $\theta$ on $M$.

For a $\theta$-slant surface, we have $\left\langle e_{1}, J e_{2}\right\rangle=\sinh \theta$. Since $\theta$-slant surfaces with $\theta=0$ are Lagrangian, we call $\theta$-slant surfaces with $\theta \neq 0$ proper slant.

The following two results from classify slant quasi-minimal surfaces in Lorentzian complex space forms $\tilde{M}_{1}^{2}(4 c)$ (see [19, Chen and Mihai]).

Theorem 14.1. There do not exist quasi-minimal proper slant surfaces in any Lorentzian complex space form $\tilde{M}_{1}^{2}(4 c)$ with $c \neq 0$.

Theorem 14.2. Let $\theta$ be a nonzero real number. Then we have:

(I) If $z(s)$ is a null curve in the light cone $\mathcal{L C}$ satisfying $\left\langle z^{\prime}, i z\right\rangle=2 \sinh \theta$, then

$$
L(s, t)=z(s) t^{\frac{1}{2}(1-i \operatorname{csch} \theta)}
$$

defines a flat quasi-minimal $\theta$-slant surface in the Lorentzian complex plane $\mathbf{C}_{1}^{2}$. 
(II) If $z(s)$ is a null curve lying in $\mathcal{L C}$ which satisfies $\left\langle z, i z^{\prime}\right\rangle=1$, then

$$
L(s, y)=z(s) e^{(i-\sinh \theta) y}
$$

defines a flat quasi-minimal $\theta$-slant surface in $\mathbf{C}_{1}^{2}$.

(III) For any given function $\varphi(t)$ defined on an open interval $I \ni 1$,

$$
\begin{aligned}
& \frac{2 \sinh \theta}{t^{\frac{1}{2}(i \operatorname{csch} \theta-1)}}\left(\int_{1}^{t} t \varphi(t) d t+t^{\frac{1}{2}(i \operatorname{csch} \theta-1)} \int_{1}^{t} \varphi(t) t^{\frac{1}{2}(3-i \operatorname{csch} \theta)} d t\right. \\
& \left.\quad-\frac{i}{2}-\frac{s}{2} \operatorname{csch} \theta, \int_{1}^{t} t \varphi(t) d t+t^{\frac{1}{2}(i \operatorname{csch} \theta-1)} \int_{1}^{t} \varphi(t) t^{\frac{1}{2}(3-i \operatorname{csch} \theta)} d t+\frac{i}{2}-\frac{s}{2} \operatorname{csch} \theta\right)
\end{aligned}
$$

defines a flat quasi-minimal $\theta$-slant surface in $\mathbf{C}_{1}^{2}$.

(IV) Let $\mu(t)$ and $\varphi(t)$ be two functions defined on an open interval $I \ni 0$. Put $F(t)=$ $\int_{0}^{t} \mu(t) d t$ and $\Phi(t)=\varphi(t) e^{-2 F(t) \sinh \theta}$. Then

$$
\begin{gathered}
\left(s e^{(i-\sinh \theta) F(t)}+(\sinh \theta-i) \int_{0}^{t} \Phi(t)\left(\int_{0}^{t} e^{(i+\sinh \theta) F(u)} d u\right) d t\right. \\
+(1+i \sinh \theta)\left(\int_{0}^{t} e^{(i+\sinh \theta) F(t)} d t\right)\left(\frac{1}{2}+i \int_{0}^{t} \Phi(t) d t\right) \\
\quad s e^{(i-\sinh \theta) F(t)}+(\sinh \theta-i) \int_{0}^{t} \Phi(t)\left(\int_{0}^{t} e^{(i+\sinh \theta) F(u)} d u\right) d t \\
\left.+(i-\sinh \theta)\left(\int_{0}^{t} e^{(i+\sinh \theta) F(t)} d t\right)\left(\frac{i}{2}+\int_{0}^{t} \Phi(t) d t\right)\right)
\end{gathered}
$$

defines a flat quasi-minimal $\theta$-slant surface in $\mathbf{C}_{1}^{2}$.

(V) Let $p(s)$ be a function defined on on open interval $I \ni 0, \phi(s, y)$ be a solution of the second differential equation

$$
\phi_{s s}-p(s) \phi=\cosh ^{2} \theta e^{-\frac{4}{3} y \sinh \theta}
$$

and $z$ be a null curve in $\mathbf{C}_{1}^{2}$ satisfying $\left\langle z^{\prime \prime}, z^{\prime \prime}\right\rangle=0$ and $\left\langle z^{\prime}, i z^{\prime \prime}\right\rangle=\cosh ^{2} \theta$. If $\phi$ is not the product of two functions of single variable, then

$$
L(s, y)=\int_{0}^{y} \frac{\phi z^{\prime \prime}-\phi_{s} z^{\prime}+\cosh ^{2} \theta e^{-\frac{4}{3} y \sinh \theta} z}{(\sinh \theta+i) e^{-y\left(i+\frac{1}{3} \sinh \theta\right)}} d y+z(s) e^{y(i-\sinh \theta)}
$$

defines a non-flat quasi-minimal $\theta$-slant surface in $\mathbf{C}_{1}^{2}$.

Conversely, quasi-minimal slant surfaces in $\mathbf{C}_{1}^{2}$ are either Lagrangian or, up to dilations and rigid motions of $\mathbf{C}_{1}^{2}$, obtained locally from the five families of proper slant surfaces. 


\section{References}

[1] J. A. Aledo, J. A. Gálvez, J. A. and P. Mira, Marginally trapped surfaces in $\mathbb{L}^{4}$ and an extended Weierstrass-Bryant representation, Ann. Global Anal. Geom. 28 (2005), 395-415.

[2] L. Andersson, M. Mars and E. Simon, Local existence of dynamical and trapping horizons, Phys. Rev. Letters 95 (2005), 111102-(1-4).

[3] A. Carrasco and M. Mars, On marginally outer trapped surfaces in stationary and static spacetimes, Classical Quantum Gravity 25 (2008), No. 5, 055011, 19.

[4] B. Y. Chen, Geometry of Slant Submanifolds, Katholieke Universiteit Leuven, Leuven, 1990.

[5] B. Y. Chen, A report on submanifolds of finite type, Soochow J. Math. 22 (1996), 117-337.

[6] B. Y. Chen, Realizations of Robertson-Walker space-times as affine hypersurfaces, J. Phys. A 40 (2007), 4241-4250.

[7] B. Y. Chen, Classification of marginally trapped surfaces, Symposium on Differential Geometry of Submanifolds, Valenciennes, France, pp.51-66, 2007, Lulu Press.

[8] B. Y. Chen, Classification of marginally trapped Lorentzian flat surfaces in $\mathbb{E}_{2}^{4}$ and its application to biharmonic surfaces, J. Math. Anal. Appl. 340 (2008), 861-875.

[9] B. Y. Chen, Minimal flat Lorentzian surfaces in Lorentzian complex space forms, Publ. Math. Debrecen, 73 (2008), 233-248.

[10] B. Y. Chen, Marginally trapped surfaces and Kaluza-Klein theory, Int. Electron. J. Geom. 2 (2009), 1-16.

[11] B. Y. Chen, Classification of spatial surfaces with parallel mean curvature vector in pseudoEuclidean spaces with arbitrary codimension, J. Math. Phys. 50 (2009), 043503, 14.

[12] B. Y. Chen, Classification of marginally trapped surfaces of constant curvature in Lorentzian complex plane, Hokkaido Math. J. 38 (2009), 361-408.

[13] B. Y. Chen, Complete classification of spatial surfaces with parallel mean curvature vector in arbitrary non-flat pseudo-Riemannian space forms, Cent. Eur. J. Math. 7 (2009), 400-428.

[14] B. Y. Chen, Complete classification of parallel spatial surfaces in pseudo-Riemannian space forms with arbitrary index and dimension, J. Geom. Phy. 60 (2010), (in press).

[15] B. Y. Chen and F. Dillen, Classification of marginally trapped Lagrangian surfaces in Lorentzian complex space forms, J. Math. Phys. 48 (2007), No. 1, 013509, 23; Erratum, J. Math. Phys. 49 (2008), No. 5, 059901.

[16] B. Y. Chen and O. J. Garay, Complete classification of quasi-minimal surfaces with parallel mean curvature vector in neutral pseudo-Euclidean 4-space $\mathbb{E}_{2}^{4}$, Results. Math. 55 (2009), 23-38.

[17] B. Y. Chen and S. Ishikawa, Biharmonic surfaces in pseudo-Euclidean spaces, Memoirs Fac. Sci. Kyushu Univ. Ser. A, Math. 45 (1991), 325-349.

[18] B. Y. Chen and S. Ishikawa, Biharmonic pseudo-Riemannian submanifolds in pseudoEuclidean spaces, Kyushu J. Math. 52 (1998), 1-18.

[19] B. Y. Chen and I. Mihai, Classification of quasi-minimal slant surfaces in Lorentzian complex space forms, Acta Math. Hungar. 122 (2009), 307-328.

[20] B. Y. Chen and J. Van der Veken, Marginally trapped surfaces in Lorentzian space forms with positive relative nullity, Classical Quantum Gravity 24 (2007), 551-563.

[21] B. Y. Chen and J. Van der Veken, Spatial and Lorentzian surfaces in Robertson-Walker space-times, J. Math. Phys. 48 (2007), No. 7, 073509, 12 
[22] B. Y. Chen and J. Van der Veken, Complete classification of parallel surfaces in Lorentzian space forms, Tohoku Math. J. 61 (2009), 1-40.

[23] B. Y. Chen and J. Van der Veken, Classification of marginally trapped surfaces with parallel mean curvature vector in Lorentzian space forms, Houston J. Math. (in press)

[24] B. Y. Chen and S. W. Wei, Differential geometry of submanifolds of warped product manifolds $I \times_{f} S^{m-1}(k)$, J. Geom. 91 (2008), 21-42.

[25] B. Y. Chen and D. Yang, Addendum to "Classification of marginally trapped Lorentzian flat surfaces in $\mathbb{E}_{2}^{4}$ and its application to biharmonic surfaces", J. Math. Anal. Appl. 361 (2010), 280-282.

[26] A. Einstein, Grundlage der allgemeinen Relativittstheorie, Ann. Phys. (Ser. IV) 51 (1916), 769-822.

[27] G. Galloway, Rigidity of marginally trapped surfaces and the topology of black holes, Comm. Anal. Geom. 16 (2008), 217-229.

[28] S. Haesen and M. Ortega, Boost invariant marginally trapped surfaces in Minkowski 4-space, Classical Quantum Gravity 24 (2007), 5441-5452.

[29] S. Haesen and L. Verstraelen, Ideally embedded space-times, J. Math. Phys. 45 (2004), 1497-1510.

[30] M. Mars and J. M. M. Senovilla, Trapped surfaces and symmetries, Classical Quantum Gravity 20 (2003), L293-L300.

[31] B. O'Neill, Semi-Riemannian Geometry with Applications to Relativity, Academic Press, New York, 1983.

[32] R. Penrose, Gravitational collapse and space-time singularities, Phys. Rev. Lett. 14 (1965), 57-59.

[33] R. Roşca, Codimension 2, CR-submanifolds with null covariant decomposable vertical distribution of a neutral manifold $\tilde{M}$, Rend. Mat. (Ser. VII) 2 (1982), 787-797.

[34] T. Sasahara, Quasi-minimal Lagrangian surfaces whose mean curvature vectors are eigenvectors, Demonstratio Math. 38 (2005), 185-196.

[35] L. Verstraelen and M. Pieters, Some immersions of Lorentz surfaces into a pseudoRiemannian space of constant curvature and of signature (2, 2), Rev. Roumaine Math. Pures Appl. 21 (1976), 593-600.

Department of Mathematics, Michigan State University, East Lansing, Michigan 48824-1027, U.S.A.

E-mail: bychen@math.msu.edu 